\title{
A novel mathematical approach to estimate the hepatic, cerebral and renal blood flow from dynamic nuclear imaging studies independently of the used radio tracer
}

\section{Barbara Katharina Geist}

Medizinische Universitat Wien

Martina Hamböck

Medizinische Universitat Wien

Neydher Berroteran-Infante

Medizinische Universitat Wien

Georg Böhmig

Technische Universitat Wien

\section{Stefanie Bugayong}

Medizinische Universitat Wien

\section{Thomas Nakuz}

Medizinische Universitat Wien

\section{Lukas Nics}

Medizinische Universitat Wien

\section{Verena Pichler}

Medizinische Universitat Wien

\section{Sazan Rasul}

Medizinische Universitat Wien

\section{Reza Agha Mohammadi Sareshgi \\ FH Campus Wien}

\section{Tatjana Traub-Weidinger}

Medizinische Universitat Wien

\section{Anna Zacher}

Medizinische Universitat Wien

\section{Anton Staudenherz}

Medizinische Universitat Wien

Marcus Hacker ( $\square$ marcus.hacker@meduniwien.ac.at )

Medical University of Vienna https://orcid.org/0000-0002-4222-4083

\section{Helmut Neufeld}

University of Vienna 
Original research

Keywords: blood flow, time activity curves, PET, scintigraphy

Posted Date: May 6th, 2020

DOI: https://doi.org/10.21203/rs.3.rs-25967/v1

License: (c) (1) This work is licensed under a Creative Commons Attribution 4.0 International License. Read Full License 


\section{Abstract \\ Background}

The blood flow (BF) is a critical determinant of organ functionality. Its assessment in the course of routine nuclear medicine examinations, including planar scintigraphy and positron emission tomography (PET), can be relevant for the diagnosis and monitoring of various conditions. The aim of this study was to investigate a new mathematical approach developed to estimate the organ BF from dynamic imaging data and to analyze if this method can be applied independent of the used radio tracer or imaging modality. The new approach uses the early phase of time activity curves extracted from animal and human dynamic scintigraphy and PET scans. Independence of tracer characteristics was evaluated with major oxygen-dependent organs (kidneys, liver, brain) of a mouse model. The approach was also applied on renal scans with two different imaging modalities from a representative cohort of 32 human subjects and compared to reference values.

\section{Results}

The mean organ-specific BF determined in the mouse model revealed no significant differences between the administered radiotracers and all calculated values corresponded to normal values (kidneys: 1.0$1.1 \mathrm{ml} / \mathrm{min}$, liver: $1.4-1.6 \mathrm{ml} / \mathrm{min}$, brain: $0.2 \mathrm{ml} / \mathrm{min}$ ). In the human study cohort, the renal BFs from the two performed imaging modalities showed a good correlation $(r=0.61, p=0.001)$ and a small significant difference $(p=0.047)$ among each other and good correlations to the reference value obtained from blood sampling $(r=0.79$ and $r=0.52)$.

\section{Conclusions}

A mathematical approach was developed to assess the organ BF solely from dynamic imaging scans without the necessity of additional measurements. Preliminary data suggests that several radiotracers might be feasible to estimate the BF in major oxygen-dependent organs.

\section{Background}

An adequate supply of oxygen and nutrients via the blood is of fundamental importance for the maintenance of the structural integrity and function of organs [1]. In healthy individuals, the organ blood flow (BF) is tightly regulated at the local level in response to varying metabolic or functional demands and remains relatively unaffected by changes in the arterial blood pressure [2]. However, many physiologic and pathophysiologic processes can cause a dysregulation of this homeostasis and result in conditions, such as portal or renovascular hypertension, with significant systemic manifestations and an increased risk for secondary organ injury $[3,4]$. Interestingly, the modification of the microvascular perfusion may also be an important mechanism of action of pharmacological interventions: sodium- 
glucose linked transporter 2 (SGLT2) inhibitors were shown to significantly reduce the effective renal plasma flow and renal BF, thus a mechanism which might contribute to the nephroprotective effects of this substance class observed in patients with diabetic kidney disease [5]. This suggests that the quantification of the $\mathrm{BF}$ to the organs might represent a yet underestimated tool to provide a more detailed insight into the pathophysiology of specific disease processes and to assess in vivo pharmaceutical effects, in particular within interventional studies.

In practice, various methods are available to determine the BF of organs. For the hepatic blood flow (HBF), methods vary from highly invasive such as plethysmography to more convenient methods such as ultrasound or magnetic resonance imaging (MRI) $[4,6,7]$. The renal blood flow (RBF), which might serve as an important parameter for the functional assessment of native and transplanted kidneys [8,9], can be determined with several minimal to non-invasive nuclear medicine methods, including planar dynamic scintigraphy and, as recently shown, also with combined positron emission tomography and MRI (PET/MRI) scans [8, 10-18]. A non-invasive, but rather sophisticated method to assess the cerebral blood flow (CBF) was described using the first minutes of a dynamic PET scan [19].

Despite of this variety of available techniques, only few studies have systematically characterized the impact of therapeutic regimes or certain pathophysiological conditions on the organ $\mathrm{BF}$, perhaps due to the limited applicability. This reinforces the need for a simple, non-invasive approach to estimate the BF in different organs in the course of routine examinations.

In this study, we describe a novel mathematical method, which was developed to estimate the organ BF by analyzing tracer kinetics in the early phase of time activity curves (TACs) extracted from dynamic imaging scans. Notably, this approach was designed to be applicable to different image modalities and tracers. Thus, it was evaluated with planar scintigraphy and PET scan data using several radiotracers: the kidney specific radiotracers [ $\left.{ }^{99 \mathrm{~m}} \mathrm{Tc}\right]$ mercaptoacetyltriglycine ([99m Tc]MAG3) and $\left[{ }^{99 \mathrm{~m}} \mathrm{Tc}\right]$ diethylenetriaminepentacetate $\left(\left[{ }^{99 \mathrm{~m}} \mathrm{Tc}\right] \mathrm{DTPA}\right)$ as well as the sugar analogues 2-deoxy-2-[$\left[{ }^{18} \mathrm{~F}\right]$ fluoro-

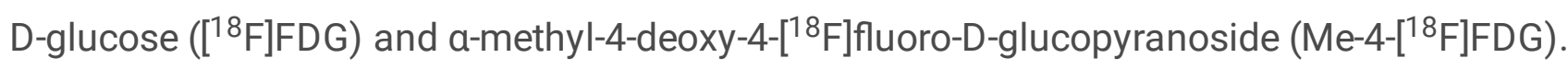

\section{Material And Methods}

Description of the new approach to determine the organ blood flow

We demonstrate a method, in which the organ $\mathrm{BF}$ is obtained by using (a) the total radiotracer activity $A(t)=V c(t)$ in the organ, where $c(t)$ is represented by the corresponding TAC and $V$ denotes the volume of the organ, as well as (b) the measured radiotracer concentration $b(t)$ in a supplying blood vessel, if possible the artery directly attached to the organ or the aorta / left ventricle, with an accordingly applied time shift. The total radiotracer activity in the organ obeys the balance equation

$\frac{d A(t)}{d t}=J_{\text {in }}(t)-J_{\text {out }}(t)$ 
where $J_{\text {in }}(t)$ refers to the radiotracer current flowing into the organ through an artery and $J_{\text {out }}(t)$ to the radiotracer current leaving the organ through a vein. We emphasize that the functions in equ. (1) should be regarded as the time average over a certain time bin length (in our case: 3 s, 5 s and 10 s, respectively), being higher than the typical pulsation period of the blood flow. As a consequence, the incoming current can be written as

$J_{\text {in }}(t)=B F \cdot b(t)$.

Assuming that the entrance of the radiotracer into the organ starts at $t=0$ (i.e. $A(t)=J_{\text {in }}(t)=0$ for $t<0$ ), integration of equ. (1) together with equ. (2) and the definition of $A(t)$ lead to the relation

$$
V c(t)=B F \cdot \int_{0}^{t} b(\tau) d \tau-\int_{0}^{t} J_{\text {out }}(\tau) d \tau=A_{\text {in }}(t)-A_{\text {out }}(t),
$$

where $A_{\text {in }}$ and $A_{\text {out }}$ can be visualized as the total number of radiotracer particles having entered or left the organ until time $\mathrm{t} ; \mathrm{Vc}(\mathrm{t})$ can be interpreted as the number of radiotracer particles present in the organ at time $t$. The first few seconds are characterized by a steep rise of the incoming current $J_{\text {in }}(t)$ followed by a subsequent decline, whereas the output current $J_{\text {out }}(t)$ starts only with some time delay at $t=t_{\text {crit }}$. Concentrating on this initial phase $\left(0<t<t_{\text {crit }}\right)$, equ. (3) with $t=t_{\text {crit }}$ allows to express $B F$ as

$$
B F=\frac{V c\left(t_{\text {crit }}\right)}{\int_{0}^{t_{c}} \text { rit } b(\tau) d \tau}
$$

being our desired relation. The only remaining task is the determination of $t_{\text {crit. }}$. Because of equ. (1), the position $t=T$ of the maximum of $A(t)$ is characterized by $J_{\text {in }}(T)=J_{\text {out }}(T)$ and we infer $0<t_{\text {crit }}<T$. Although we have no direct information on the output current itself, the turning point of $A(t)$ before its maximum serves as an excellent estimate for the value of $t_{\text {crit }}$.

In other words, it is assumed that the total number of radiotracer particles entering an organ via the $\mathrm{BF}$ must be equal to the total number of particles in the organ $\mathrm{Vc}(\mathrm{t})$ until $\mathrm{t}_{c r i t}$ is reached. With a precise determination of the volume $V$ and a sufficient time resolution in the measurement of the TACs $b(t)$ and $\mathrm{c}(\mathrm{t})$, the $\mathrm{BF}$ can be determined by simply finding the turning point of $\mathrm{c}(\mathrm{t})$ and inserting all values into equ. (4).

In Fig. 1, typical total TACs (i.e. Vc(t)) of the kidneys with three radiotracers are shown (gray curves), as well as the according $A_{\text {in }}(t)$ with the BF calculated from equ. (4). $t_{\text {crit }}$ was taken as the turning point of the rising total TAC, which is indicated with an arrow in the small images.

In the following, the hereby determined BF is denoted as RBF-new-MAG3, RBF-new-FDG, RBF-new-DTPA and RBF-new-Me4 for the renal blood flow, calculated for the radiotracers $\left[{ }^{99 \mathrm{~m}} \mathrm{Tc}\right] \mathrm{MAG},\left[{ }^{18} \mathrm{~F}\right] \mathrm{FDG}$, $\left[{ }^{99 m}\right.$ TC]DTPA and Me-4- $\left[{ }^{18}\right.$ F]FDG, respectively. The obtained single values for the left and right kidney 
were summed up. The cerebral and hepatic blood flows is denoted as CBF-new-FDG and CBF-new-Me4, as well as HBF-new-FDG and HBF-new-Me4, respectively.

\section{Radiotracer production}

Fully-automated radiopharmaceutical production and quality control of $\left[{ }^{18} \mathrm{~F}\right] \mathrm{FDG}$ and investigational Me$4-\left[{ }^{18} \mathrm{~F}\right] F D G$ were performed on a commercially available synthesizer GE Fastlab at the PET centre of the General Hospital Vienna. GMP-grade precursor and reference standard for Me-4-[ $\left.{ }^{18} \mathrm{~F}\right] \mathrm{FDG}$ were obtained by ABX (Radeberg, Germany). Commercially available kits for the preparation of [ ${ }^{99 \mathrm{~m}} \mathrm{Tc}$ ]MAG3 (Mertioscan) were distributed by DSD Pharma (Purkersdorf, Austria) and for the preparation of $\left[{ }^{99 \mathrm{~m}}\right.$ Tc]DTPA (TechneScan) by BSM diagnostic (Vienna, Austria).

Animal study design and scan protocols

The mouse study (ethics application number BMBWF-66.009/0144-V/3b/2018) was performed at the Preclinical Imaging Laboratory at the Division of Nuclear Medicine, Medical University of Vienna, and included 7 healthy male C57BLKS mice. All mice were examined with a planar dynamic scintigraphy with the radio radiotracer $\left[{ }^{99 \mathrm{~m}} \mathrm{Tc}\right] \mathrm{DTPA}$. One day later, mice were scanned with an additional dynamic combined PET and computer tomography (PET/CT) scan, four of them with $\left[{ }^{18} \mathrm{~F}\right] \mathrm{FDG}$ and three with Me$4-\left[{ }^{18} \mathrm{~F}\right] \mathrm{FDG}$.

During all scans, mice were kept under anesthesia with a mixture of isoflurane (2\%) and $\mathrm{O}_{2}(2.5 \mathrm{I} / \mathrm{min})$ continuously administered via a mask and kept at a body temperature of $37^{\circ} \mathrm{C}$. The heart rate and breathing frequency were monitored. Anaesthetized animals were positioned in the animal Inveon PET/SPECT/CT scanner (Siemens Medical Solutions, Knoxville, TN) and the according radiotracer was injected via the lateral tail vein. All dynamic scans started with the injection and lasted for $45 \mathrm{~min}$. For the PET scans, an additional CT image was obtained and used for attenuation correction and co-registration. The latter were reconstructed by Fourier re-binning followed by 2-dimensional filtered back projection with a ramp filter. CT raw data were reconstructed with a Feldkamp algorithm using a Shepp-Logan filter followed by standard mouse beam-hardening correction and noise reduction (matrix size: $1024 \times 1024$; effective pixel size: $97.56 \mu \mathrm{m}$ ). The standard data correction protocol (normalization, attenuation, decay correction and injection decay correction) was applied to the PET data. All dynamic data sets were rebinned into a dynamic sequence of $8 \times 3 \mathrm{~s}, 9 \times 5 \mathrm{~s}, 13 \times 10 \mathrm{~s}$ and $11 \times 222 \mathrm{~s}$. Volumes of interests (VOls) of the liver, the brain, the kidneys and the aorta were delineated by hand on the CT images and transferred to the individual time frame PET images using PMOD software (version 3.8, Pmod Ltd, Zurich, Switzerland). With the same software, regions of interest (ROIs) were delineated in the planar scintigraphy images around the kidneys, the perirenal background and the heart.

The normal values for renal blood flow (RBF), hepatic blood flow (HBF) and cerebral blood flow (CBF) were compared to normal values found in literature [20-23] accounting for RBF-norm with $0.8 \mathrm{ml} / \mathrm{min}$, 
HBF-norm with $1.8 \mathrm{ml} / \mathrm{min}$ and $\mathrm{CBF}$-norm around $0.26 \mathrm{ml} / \mathrm{min}$. They are denoted as RBF-norm, HBF-norm and CBF-norm

Human study design and scan protocols

The human data set (ethics no. 1068/2015 and 1734/2017) included 32 adult subjects with normal and impaired renal function. Subjects underwent a dynamic (PET/MRI) scan with the radiotracer $\left[{ }^{18} \mathrm{~F}\right] \mathrm{FDG}$, and around 2 weeks earlier or later a dynamic planar renal scintigraphy with the radiotracer [ $\left.{ }^{99 \mathrm{~m}} \mathrm{Tc}\right] \mathrm{MAG} 3$.

All subjects were hydrated with water $(10 \mathrm{ml} / \mathrm{kg}$ body weight) $20 \mathrm{~min}$ prior to the examination and asked to empty their bladder directly before injection of the according radiotracer. Starting with an injection of around $3 \mathrm{MBq} / \mathrm{kg}$ body weight of $\left[{ }^{18} \mathrm{~F}\right] \mathrm{FDG}$, the dynamic PET/MRI scans (Siemens Biograph mMR, Siemens Healthcare Diagnostics $\mathrm{GmbH}$, Germany) were performed for 30 minutes. The PET reconstructed (Siemens e7 tools) list-mode data were re-binned into a dynamic sequence of $60 \times 5 \mathrm{~s}, 25 \times$ 60 s, with $172 \times 172 \times 127$ matrix, using ordinary Poisson ordered subset expectation maximization (OPOSEM) 3D algorithm ( 3 iterations, 21 subsets, Gaussian filter) and Dixon-based MRI-attenuation. The MRI imaging protocol consisted of a T1 weighted MRI sequence (axial breath holding and fat suppression, VIBE SPAIR). In the fused images, three VOIs were delineated by hand using the Hermes Hybrid Viewer tool (Hermes Medical Solutions AB, Stockholm, Sweden): aorta descendens, left and right kidney (see fig. 2).

The planar scintigraphy scans were performed according to the EANM guidelines [23] after the injection of $80 \mathrm{MBq}$ [ $\left.{ }^{99 \mathrm{~m}} \mathrm{Tc}\right] \mathrm{MAG} 3$ and lasted for 20 minutes. The images were acquired with a large field of view gamma camera (low-energy, high-resolution collimator, $64 \times 64$ matrix, frame rate $10 \mathrm{~s} /$ frame, energy window $140 \mathrm{keV}$ with $20 \%$ width). In case of 24 subjects, a standard was additionally measured with the gamma camera for 10 seconds. Using Hermes software, ROls were drawn around the kidneys, the perirenal background (see fig. 1) as well as in the left ventricle.

From all subjects, one blood sample was drawn before the first scan and used to determine the hematocrit value (Hct); a second blood sample was drawn $41 \pm 2$ minutes after each scintigraphy, which was measured, together with a standard, in a gamma counter.

For the human data set, two reference values were available. The blood-sample derived method RBFblood, originally developed by Tauxe et al. [10], is based on the renal clearance of a radiotracer by measuring drawn blood samples after its venous injection together with a standard in a gamma counter. In this study, the effective renal plasma flow (eRPF) was determined from the [ $\left.{ }^{99 \mathrm{~m}} \mathrm{Tc}\right] \mathrm{MAG} 3$ clearance according to a refined method by Russell et al. [8,12], from which RBF-blood was then calculated by dividing eRPF by $(1-\mathrm{Hct})$.

The image derived, graphical methods are based on the idea that the renal $\left[{ }^{99 m} \mathrm{Tc}\right] \mathrm{MAG} 3$ uptake within the first few minutes can be converted to the eRPF provided, also a standard is measured. The graphical eRPF was calculated for each kidney from the renal uptake between minute 2 and 3 after injection 
according to the formula of Arroyo et al. [12,13]. The resulting eRPF was divided by (1- Hct) and summed for both kidneys in order to obtain RBF-graph. Note that in case of 8 subjects, no standard was measured with the gamma camera, omitting the determination of RBF-graph. All human RBF values were normalized to a body surface are of $1.73 \mathrm{~m}^{2}$.

A summary of all available data sets is given in table 1.

Table 1

Summary of all available data sets from dynamic imaging scans. CT: computed tomography; MRI: magnetic resonance imaging; PET: positron emission tomography; CBF: cerebral blood flow; HBF: hepatic blood flow; RBF: renal blood flow;

\begin{tabular}{|c|c|c|c|c|c|}
\hline $\begin{array}{l}\text { Data } \\
\text { set }\end{array}$ & $\begin{array}{l}\text { cohort } \\
\text { size }\end{array}$ & $\begin{array}{l}\text { Investigated } \\
\text { organs }\end{array}$ & $\begin{array}{l}\text { Examination } \\
\text { methods }\end{array}$ & $\begin{array}{l}\text { According } \\
\text { radiotracers }\end{array}$ & $\begin{array}{l}\text { According determined } \\
\text { blood flows }\end{array}$ \\
\hline Human & 32 & kidneys & $\begin{array}{l}\text { PET/MRI } \\
\text { scintigraphy } \\
\text { blood samples }\end{array}$ & $\begin{array}{l}{\left[{ }^{18} \mathrm{~F}\right] \mathrm{FDG}} \\
{\left[{ }^{99 \mathrm{~m}} \mathrm{Tc}\right] \mathrm{MAG} 3}\end{array}$ & $\begin{array}{l}\text { RBF-new-FDG } \\
\text { RBF-graph, RBF-new- } \\
\text { MAG3 } \\
\text { RBF-blood }\end{array}$ \\
\hline Mouse & 7 & kidneys & $\begin{array}{l}\text { PET/CT } \\
\text { scintigraphy }\end{array}$ & $\begin{array}{l}{\left[{ }^{18} \mathrm{~F}\right] \mathrm{FDG}, \mathrm{Me}-4-} \\
{\left[{ }^{18} \mathrm{~F}\right] \mathrm{FDG}} \\
{\left[{ }^{99 \mathrm{~m}} \mathrm{Tc}\right] \mathrm{DTPA}}\end{array}$ & $\begin{array}{l}\text { RBF-new-FDG, RBF-new- } \\
\text { Me4 } \\
\text { RBF-new-DTPA }\end{array}$ \\
\hline & & liver & $\mathrm{PET} / \mathrm{CT}$ & $\begin{array}{l}{\left[{ }^{18} \mathrm{~F}\right] \mathrm{FDG}, \mathrm{Me}-4-} \\
{\left[{ }^{18} \mathrm{~F}\right] \mathrm{FDG}}\end{array}$ & $\begin{array}{l}\text { HBF-new-FDG, HBF- } \\
\text { new-Me4 }\end{array}$ \\
\hline & & brain & $\mathrm{PET} / \mathrm{CT}$ & $\begin{array}{l}{\left[{ }^{18} \mathrm{~F}\right] F D G, M e-4-} \\
{\left[{ }^{18} \mathrm{~F}\right] F D G}\end{array}$ & $\begin{array}{l}\text { CBF-new-FDG, CBF-new- } \\
\text { Me4 }\end{array}$ \\
\hline
\end{tabular}

Time activity curves and organ volumes

The radiotracer concentrations over time, i.e. the time activity curves (TACs) of all animal and human scans were exported in units of kilobecquerel per milliliter $(\mathrm{kBq} / \mathrm{ml})$. TACs from planar scintigraphy images were corrected for the perirenal background. Via linear interpolation between the measured concentration points, the time binning of the TACs was reduced to one second. The organ TACs were smoothed with a Savitzky-Golay filter, the TACs from the blood pool (heart or aorta) were fitted with a three-exponential curve starting from their according peak.

For human PET/MRI data, kidney volumes were measured using the MRI sequences. Animal organ volumes from the PET data were measured in the co-registered CT images.

In order to assess the renal volumes from the measured kidney areas in the images from the human and animal renal planar scintigraphy, an isotropic scaling was performed, i.e. a transformation of an area to a volume by multiplying the area with its square root and an appropriate factor. For humans, the kidney 
was considered as an ellipsoid with an averaged thickness between 4 and $5 \mathrm{~cm}$ and a height of 11 to $13 \mathrm{~cm}$. Therefore, the appropriate factor was set to 0.4 . For mice, the same renal dimension ratios were assumed, resulting in the same factor.

Statistical analysis

Mean, minimum and maximum values were calculated for each BF value. For the comparison of the blood flow of a certain organ between different radiotracers, an unpaired Student's t-test was performed. For comparisons between new and reference values of the same organ, the Pearson correlation coefficient $r$ and the Student's paired t-test was applied. A p value $<0.05$ was considered as statistically significant. All according calculations were performed with LibreOffice version 5.3.7.2.

\section{Results}

All obtained BF mean values and ranges from minimum to maximum are summarized in Table 2. A summary of human subject demographics (female: $n=10$ female, male: $n=22$ ) is shown in Table 3 .

Table 2

Mean values (ranges in parenthesis) of all blood flow (BF) values: renal blood flow (RBF), hepatic blood flow (HBF) and cerebral blood flow (CBF). The epithet "new" indicated that the according BF was calculated with the new approach. The normal values, indicated with the epithet "norm", were taken from refs. [20-23].

\begin{tabular}{|llll|}
\hline & organ & According BF & Mean value (range) [ml/min] \\
\hline animal & kidney & RBF-new-FDG & $1.0(0.7-1.4)$ \\
& & RBF-new-Me4 & $1.1(0.3-2.0)$ \\
& & RBF-new-DTPA & $1.0(0.5-1.4)$ \\
& & RBF-norm & $0.8-1.3$ \\
\hline \multirow{2}{*}{ liver } & HBF-new-FDG & $1.6(1.4-1.7)$ \\
& & HBF-new-Me4 & $1.4(0.7-2.1)$ \\
& & HBF-norm & 1.8 \\
\hline \multirow{2}{*}{ brain } & CBF-new-FDG & $0.2(0.12-0.18)$ \\
& & CBF-new-Me4 & $0.2(0.17-0.24)$ \\
& & CBF norm & 0.26 \\
human & kidney & RBF-blood & $1070(477-2025)$ \\
& & RBF-graph & $938(468-1402)$ \\
& RBF-new-FDG & $914(297-1717)$ \\
& RBF-new-MAG3 & $1074(52-2154)$ \\
\hline
\end{tabular}


Table 3

Summary of patient demographics.

\begin{tabular}{|llllll|}
\hline & $\begin{array}{l}\text { Age } \\
\text { [years] }\end{array}$ & $\begin{array}{l}\text { Height } \\
{[\mathbf{c m}]}\end{array}$ & $\begin{array}{l}\text { Weight } \\
{[\mathrm{kg}]}\end{array}$ & $\begin{array}{l}\text { Hematocrit } \\
{[\%]}\end{array}$ & $\begin{array}{l}\text { Glomerular filtration rate } \\
{[\mathrm{ml} / \mathrm{min}]}\end{array}$ \\
\hline $\begin{array}{l}\text { Mean } \pm \text { standard } \\
\text { deviation }\end{array}$ & $43 \pm 15$ & $\begin{array}{l}179 \pm \\
10\end{array}$ & $86 \pm 18$ & $42.5 \pm 3.9$ & $119 \pm 36.3$ \\
\hline $\begin{array}{l}\text { Range (minimum - } \\
\text { maximum) }\end{array}$ & $21-74$ & $\begin{array}{l}160- \\
200\end{array}$ & $50-120$ & $34.8-48.9$ & $13-242$ \\
\hline
\end{tabular}

Comparisons between different radiotracers

For animal kidney data, RBF-new-FDG, RBF-new-Me4 and RBF-new-DTPA were obtained. There was no significant difference found, neither between RBF-new-FDG and RBF-new-Me4 (10\% difference, $p=0.84$ ), nor between RBF-new-FDG and RBF-new-DTPA (4\% difference, $p=0.96$ ), nor between RBF-new-Me4 and RBF-new-DTPA (12\% difference, $p=0.78$ ). In case of the liver, HBF-new-FDG and HBF-new-Me4 were compared, showing no significant difference between each other ( $11 \%$ difference, $p=0.64)$. Also, in case of mouse brain data, no significant difference was found between CBF-new-FDG and CBF-new-Me4 (31\% difference, $p=0.09$ ).

From human kidney data (human data set, see Table 1), RBF-new was calculated for the radiotracers $\left[{ }^{18} \mathrm{~F}\right] F D G$ and $\left[{ }^{99 m}\right.$ Tc]MAG3, i.e. RBF-new-FDG and RBF-new-MAG3 were obtained according to equ. 2. A correlation of $r=0.61(p=0.001)$ was found between them, with a by $18 \%$ significantly lower RBF-newFDG mean value $(p=0.047)$.

Comparison to reference values

For animal data, all RBF values (RBF-new-FDG, RBF-new-Me4 and RBF-new-DTPA) were in excellent agreement with normal RBF values (0.8 to $1.1 \mathrm{ml} / \mathrm{min}$ ). The liver values, HBF-new-FDG and HBF-new-Me4 were slightly lower than the normal values ( 1.6 and 1.4 versus $1.8 \mathrm{ml} / \mathrm{min}$, respectively). Similarly, also brain values CBF-new-FDG and CBF-new-Me4 were with averaged $0.2 \mathrm{ml} / \mathrm{min}$ slightly lower than norm values $(0.26 \mathrm{ml} / \mathrm{min})$.

In case of human kidney data, reference values RBF-blood and RBF-graph were available. Both RBF-newFDG and RBF-new-MAG3 had good correlations to both reference methods (see Table 4 and Fig. 3). RBFnew-FDG showed significant differences to RBF-blood, but not to RBF-graph, which was opposite for RBFnew-MAG3. Note that both reference methods, RBF-blood and RBF-graph, also showed a significant difference of $12 \%$ and a correlation of $r=0.57$ between each other. 
Table 4

Comparison of renal blood flow (RBF) obtained with the new method from two radiotracers $\left(\left[{ }^{18} \mathrm{~F}\right] \mathrm{FDG}\right.$ and $\left.\left[{ }^{99 \mathrm{~m}} \mathrm{TC}\right] \mathrm{MAG} 3\right)$ with two reference methods obtained from

blood sample measurements (RBF-blood) and with an image derived graphical method (RBF-graph): correlation coefficient $r$ (with according $p$ value) and difference in percent (with according $\mathrm{p}$ value).

\begin{tabular}{|llll|}
\hline & RBF-new-MAG3 & RBF-blood & RBF-graph \\
\hline RBF-new-FDG & $r=0.61(p=0.001)$ & $r=0.79(p<0.001)$ & $r=0.49(p=0.02)$ \\
& $d=18 \%(p=0.047)$ & $d=15 \%(p=0.001)$ & $d=3 \%(p=0.51)$ \\
\hline RBF-new-MAG3 & & $r=0.52(p=0.008)$ & $r=0.53(p=0.007)$ \\
& & $d=0 \%(p=0.94)$ & $d=15 \%(p=0.04)$ \\
RBF-blood & & & $r=0.57(p=0.003)$ \\
& & & $d=12 \%(p=0.03)$ \\
\hline
\end{tabular}

\section{Discussion}

A comprehensive mathematical treatise about the estimation of the organ blood flow independent of the radiotracer or the organ was already done by Bassingthwaighte and Holloway [23], based on the measurement of both arterial inflow and venous outflow, whereas the latter is hampered by an insufficient determination of the venous radiotracer concentration in functional imaging data. Using solely the inflow and the measured tissue concentration, a solution was presented for the CBF by Raichele et al. [19], which starts with similar consideration as our approach, but is more sophisticated and dependent on a not analytically solvable integration. While many methods for the blood flow determination of organs are invasive or impracticable, several non-invasive methods based on imaging modalities were developed. However, most of them are specific to an organ of interest or a radiotracer, e.g. using certain MRI sequences for the HBF [7], dynamic contrast enhanced CT measurements for the CBF [26] or image derived, graphical methods from scintigraphy images for the RBF [14-17].

In this study, we evaluated a new approach allowing to determine theoretically any organ blood flow from dynamic imaging scans independently of the used radiotracer, organ or modality. The presented approach uses the early phase of dynamic imaging scans and is non-invasive, provided the dynamic scan is reconstructed with a sufficient high time binning and the blood pool concentration can be measured in the obtained images, respectively. The latter is possible if the field of view of the scanner covers the upper part of the aorta, which was shown to have minor partial volume and motion effects [18, 27]. In case of planar scintigraphy, performed e.g. for a renal scan, the blood concentration usually can be measured from the heart, since the according radiotracers show no uptake in the myocardium.

The reliability of the new approach was first evaluated first on PET/CT and scintigraphy scans of animals in three major oxygen-dependent organs. The results support our assumption that the early phase of a 
dynamic scan is solely dependent on the organ blood flow and not on the radiotracer pharmacokinetics, since the hereby calculated renal blood flow RBF was almost equal between three different radiotracers $\left[{ }^{18} \mathrm{~F}\right] F D G, M e-4-\left[{ }^{18} \mathrm{~F}\right] F D G$ and $\left[{ }^{99 m}\right.$ Tc]DTPA and in excellent agreement with normal values. Also the HBF showed no difference between the two available radiotracers Me-4-[ $\left[{ }^{18} \mathrm{~F}\right] \mathrm{FDG}$ and $\left[{ }^{18} \mathrm{~F}\right] \mathrm{FDG}$. The small difference to the normal value might be due to the fact, that only the arterial influx to the liver was used for HBF calculation and the contribution of the portal vein was ignored. More impressive, in contrary to $\left[{ }^{18} \mathrm{~F}\right] \mathrm{FDG}$, the radiotracer Me-4-[18 F]FDG barely passes the blood brain barrier [28] resulting in a very low cerebral uptake, but again no significant differences between $\left[{ }^{18} \mathrm{~F}\right] \mathrm{FDG}$ and Me-4-[ $\left.{ }^{18} \mathrm{~F}\right] \mathrm{FDG}$ could be found in the CBF (both with a CBF of averaged $0.2 \mathrm{ml} / \mathrm{min}$ with $31 \%$ difference, $p=0.09$ ), although both CBF estimations were slightly lower compared to the normal value.

Similar results were obtained from human renal data, where the new approach to calculate the RBF was applied on one cohort investigated with two different radiotracers and modalities: the sugar analogue $\left[{ }^{18} \mathrm{~F}\right] \mathrm{FDG}$ using PET/MRI scans and the kidney specific radiotracer $\left[{ }^{99 \mathrm{~m}} \mathrm{Tc}\right] \mathrm{MAG} 3$ using scintigraphy. There was only a slight significant difference between both RBF-new-MAG3 and RBF-new-FDG $(p=0.047)$ and they showed a good correlation $(r=0.61)$. In comparison with the two available reference methods, RBF-new-FDG correlated well with the blood sample reference method and worse (although still significant) with the graphical one, while showing a significant difference to the first and a small difference to the latter; this result was opposite for the RBF-new-MAG3. Interestingly, the two reference methods showed among each other a significant but surprisingly low correlation of $r=0.57$ and a significant difference of $12 \%(p=0.03)$. Using a radiotracer similar to [99mTc]MAG3, Fine et al [29] also compared a graphical method to a blood sample derived for calculating the renal plasma flow, obtaining much better correlations of $r=0.90$, which is most certainly related to their wider range of available values. However, they also observed a systematic difference between both methods.

Concerning this study, it is mentioned that there exist four main differences between the scanning protocols of $\left[{ }^{99 \mathrm{~m}} \mathrm{Tc}\right] \mathrm{MAG} 3$ and $\left[{ }^{18} \mathrm{~F}\right] \mathrm{FDG}$. First, the reconstruction protocol of the planar [ $\left.{ }^{99 \mathrm{~m}} \mathrm{Tc}\right] \mathrm{MAG} 3$ scans used higher time frames of $10 \mathrm{sec}$, leading to a probably insufficient low number of data points in the early phase used for RBF. Second, planar imaging methods need a background subtraction leading to errors [30]. Third, no accompanying imaging method for anatomical information was performed, making the delineation of the heart precarious. Finally, the new approach relies on a determination of organ volumes, which needs to be estimated from planar areas in case of renal scintigraphy, which we did with an isotropic scaling. The isotropic scaling obviously leads to negligible differences in case of the planar animal scintigraphy data using genetically almost identical mice, but most certainly not for the human data. However, RBF-new-MAG3 and the reference method RBF-graph showed very similar differences and correlations to the probably most reliable reference method RBF-blood, thus RBF-new-MAG3 might be considered as a comparable convenient method to determine the RBF from planar renal scintigraphy, with the additional advantage of omitting a standard measurement.

\section{Limitations}


The blood TACs were image derived and not corrected for partial volume or motion effects. Although these effects have probably a minor impact when derived from the aorta, omitting an appropriate correction might also explain deviations to reference or normal values. Also, no reference methods were available for the animal data. However, a further separate according animal study would have been beyond of the scope of this proof-of-concept paper.

Furthermore, this study not nearly covers all available imaging methods, organs or radiotracers. The new approach therefore needs further investigation in other settings, which will be done in our clinic in the course of future dynamic studies.

With the presented new approach, in principle a scan lasting for a few minutes might be sufficient to determine an organ blood flow. While the clinical practicability of such a short scan is questionable, the new approach might be used in the course of dynamic scans allowing an additional assessment of those organ blood flows which are in the field of view.

\section{Conclusions}

A new mathematical approach was presented using the early phase of dynamical functional imaging. Provided the blood and tissue concentrations were obtained with sufficient time resolution, it was shown that this approach allows to asses at least the renal, hepatic and cerebral blood flow independently of the administered tracer or imaging modality, and being in good agreement with normal and reference values.

\section{Abbreviations}

$\mathrm{BF}$

blood flow

CBF

cerebral blood flow

CT

computer tomography

$\left[{ }^{18} \mathrm{~F}\right] \mathrm{FDG}$

2-deoxy-2-[18 F]fluoro-D-glucose

$\mathrm{HBF}$

hepatic blood flow

[99m Tc]MAG3

[ $\left.{ }^{99 \mathrm{~m}} \mathrm{Tc}\right]-\mathrm{mercaptoacety}$ ltriglycine

[99m Tc]DTPA

$\left[{ }^{99 \mathrm{~m}} \mathrm{Tc}\right]-$ diethylenetriaminepentacetate

Me-4-[ ${ }^{18}$ F]FDG

a-methyl-4-deoxy-4-[ ${ }^{18}$ F]fluoro-D-glucopyranoside 
MRI

magnetic resonance imaging

PET

positron emission tomography

RBF

renal blood flow

\section{Declarations}

Ethics approval: All studies (1068/2015, 1734/2017 and BMBWF-66.009/0144-V/3b/2018) were approved by the ethics committee of the Medical University of Vienna.

Consent for publication: not applicable.

Availability of data and material: The datasets generated and/or analysed during the current study are not publicly available due to the size of dynamic scan data, but are available from the corresponding author on reasonable request.

Competing interests: The authors declare that they have no competing interests.

Funding: not applicable.

Author's contributions: BKG was involved in method creation and drafted the manuscript, organized the studies and evaluated all data. MH drafted the manuscript and was in charge of patient treatment. NBI and VP were in charge of tracer production. GB gave input to the clinical part. SB, AZ and LN were in charge of animal scans. TN, SR, RAMS, TTW and AS performed the human studies. MH designed all studies and edited the manuscript. HN developed the method and drafted the manuscript.

Acknowledgments: Many thanks to Fritz Gierschele for his work in the tracer production.

\section{References}

1. Jayanthy AK, Sujatha N, Ramasubba Reddy M. Measuring blood flow: techniques and applications a review. J Radiat Red Appl Sc. 2011;6(2):203-16.

2. Secomb TW. Theoretical Models for Regulation of Blood Flow. Microcirculation. 2008;15(8):765-75.

3. Herrmann SM, Textor SC. Current Concepts in the Treatment of Renovascular Hypertension. Am J Hypertens. 2018;31(2):139-49.

4. Johnson DJ, Muhlbacher F, Wilmore DW. Measurement of Hepatic Blood Flow. J Surg Res. 1985;39:470-81.

5. Cherney DZ, Perkins BA, Soleymanlou N, Maione M, Lai V, Lee A, Fagan NM, Woerle HJ, Johansen OE, Broedl UC, con Eynatten M. Renal hemodynamic effect of sodium-glucose cotransporter 2 inhibition in patients with type 1 diabetes mellitus. Circulation. 2014;4(5):587-97. 129(. 
6. Seifalain AM, Stansby GP, Hobbs KEF, Hawkes DJ, Colchester ACF. Measurement of liver blood flow: a review. HPB Surg. 1991;4:171-86.

7. Dyvorne HA, Knight-Greenfield A, Besa C, Cooper N, Garcia-Flores J, Schiano TD, Taouli B. Quantification of Hepatic Blood Flow Using a High-Resolution Phase-Contrast MRI Sequence with Compressed Sensing Acceleration. AJR Am J Roentgenol. 2015;204(3):510-8.

8. Russell CD, Taylor A, Eshima D. Estimation of Technetium-99m-MAG3 Plasma Clearance in Adults from One or Two Blood Samples. J Nuc Med. 1989;30:1955-9.

9. Dubovsky EV, Logic JR, Diethelm AG, Balch CM, Tauxe WN. Comprehensive evaluation of renal function in the transplanted kidney. J Nuc Med. 1975;16(12):1115-20.

10. Tauxe WN, Maher FT, Taylor WF. Effective renal plasma flow: estimation from theoretical volumes of distribution of intravenously injected 1311-orthoiodohippurate. Mayo Clin Proc. 1971;46:524-31.

11. Bubeck B, Piepenburg R, Grethe U, Ehrig B, Hahn K. A new principle to normalize plasma concentrations allowing single-sample clearance determinations in both children and adults. Eur $\mathrm{J}$ Nucl Med. 1992;19:511-6.

12. Russel CD, Taylor AT, Dubovsky EV. Measurement of Renal Function with Technetium-99m-MAG3 in Children and Adults. J Nuc Med. 1996;37(4):588-93.

13. Schlegel JU, Hamway SA. Individual renal plasma flow determination in 2 minutes. J Urol. 1978;116:282-4.

14. Schlegel JU, Halikiopoulus HL, Prima R. Determination of filtration fraction using the gamma scintillation camera. J Urol. 1978;122:447-50.

15. Arroyo AJ. Comparison of Technetium-99m MAG3 and lodine-131 OIH ERPF Results Using the Camera Technique. J Nucl Med Technol. 1991;19(3):173-5.

16. Arroyo JA. Effective Renal Plasma Flow Determination Using Technetium-99m MAG3: Comparison of Two Camera Techniques with the Tauxe Methods. J Nucl Med Technol. 1993;21:163-6.

17. Taylor A, Corrigan PL, Galt J, Garcia EV, Folks R, Jones M, et al. Measuring Technetium-99m-MAG3 Clearance with an Improved Camera-Based Method. J Nuc Med. 1995;36:1689-95.

18. Geist BK, Baltzer P, Fueger B, et al. Assessing the kidney function parameters glomerular filtration rate and effective renal plasma flow with dynamic FDG-PET/MRI in healthy subjects. Eur $\mathrm{J}$ Nucl Med Mol I Res. 2018;8:37.

19. Raichle ME, Martin WRW, Herscovltch P, Mintun MA, Markham J. Brain blood flow measured with intravenous H2150 II Implementation and Validation. J Nucl Med. 1983;24:790-8.

20. Davies B, Morris T. Physiological Parameters in Laboratory Animals and Humans. Pharm Res. 1993;10:1093-5.

21. Iliescu R, Cazan R, McLemore GR, Venegas-Pont M, Ryan MJ. Renal blood flow and dynamic autoregulation in conscious mice. Am J Physiol Renal Physiol. 2008;295(3):F734-40.

22. Hall $C$, Lueshen $E$, Mošat A, Linninger AA. Interspecies scaling in pharmacokinetics: a novel wholebody physiologically based modeling framework to discover drug biodistribution mechanisms in 
vivo. J Pharm Sci. 2012;101(3):1221-41.

23. Muir ER, Shen Q, Duong TQ. Cerebral Blood Flow MRI in Mice using the Cardiac-Spin-Labeling Technique. Magn Reson Med. 2008;60(3):744-8.

24. Taylor AT, Brandon DC, de Palma D, Blaufox MD, Durand E, Erbas B, Grant SF, Hilson AJW, Morsing A. SNMMI Procedure Standard/EANM PracticeGuideline for Diuretic Renal Scintigraphy in Adults With Suspected Upper Urinary Tract Obstruction 1.0. Sem Nucl Med. 2018;48(4):377-90.

25. Bassingthwaighte JB, Holloway GA. Estimation of Blood Flow With Radioactive Tracers. Sem Nucl Med. 1976;6(2):141-61.

26. Cenic A, Nabavi DG, Craen RA, Gelb AW, Lee TY. A CT Method to Measure Hemodynamics in Brain Tumors: Validation and Application of Cerebral Blood Flow Maps. Am J Neuroradiology. 2000;21(3):462-70.

27. Germano G, Chen BC, Huang SC, Gambhir SS, Hoffman EJ, Phelps ME. Use of the abdominal aorta for arterial input function determination in hepatic and renal PET studies. J Nucl Med. 1992;33:61320.

28. Yu AS, Hirayama BA, Timbol G, Liu J, Basarah E, Kepe V, Satyamurthy S, Huang SH, Wright EM, Barrio JR. Functional expression of SGLTs in rat brain. Am J Physiol Cell Physiol. 2010;299(6):C1277-84.

29. Fine EJ, Axelrod M, Gorkin J, Saleemi K, Blaufox MD. Measurement of Effective Renal Plasma Flow: A Comparison of Methods. J Nucl Med. 1987;28:1393-400.

30. Geist BK, Dobrozemsky G, Samal M, Schaffarich MP, Sinzinger H. Staudenherz A WWSSF-a worldwide study on radioisotopic renal split function: reproducibility of renal split function assessment in children. Nucl Med Commun. 2015;36(12):1233-8.

\section{Figures}



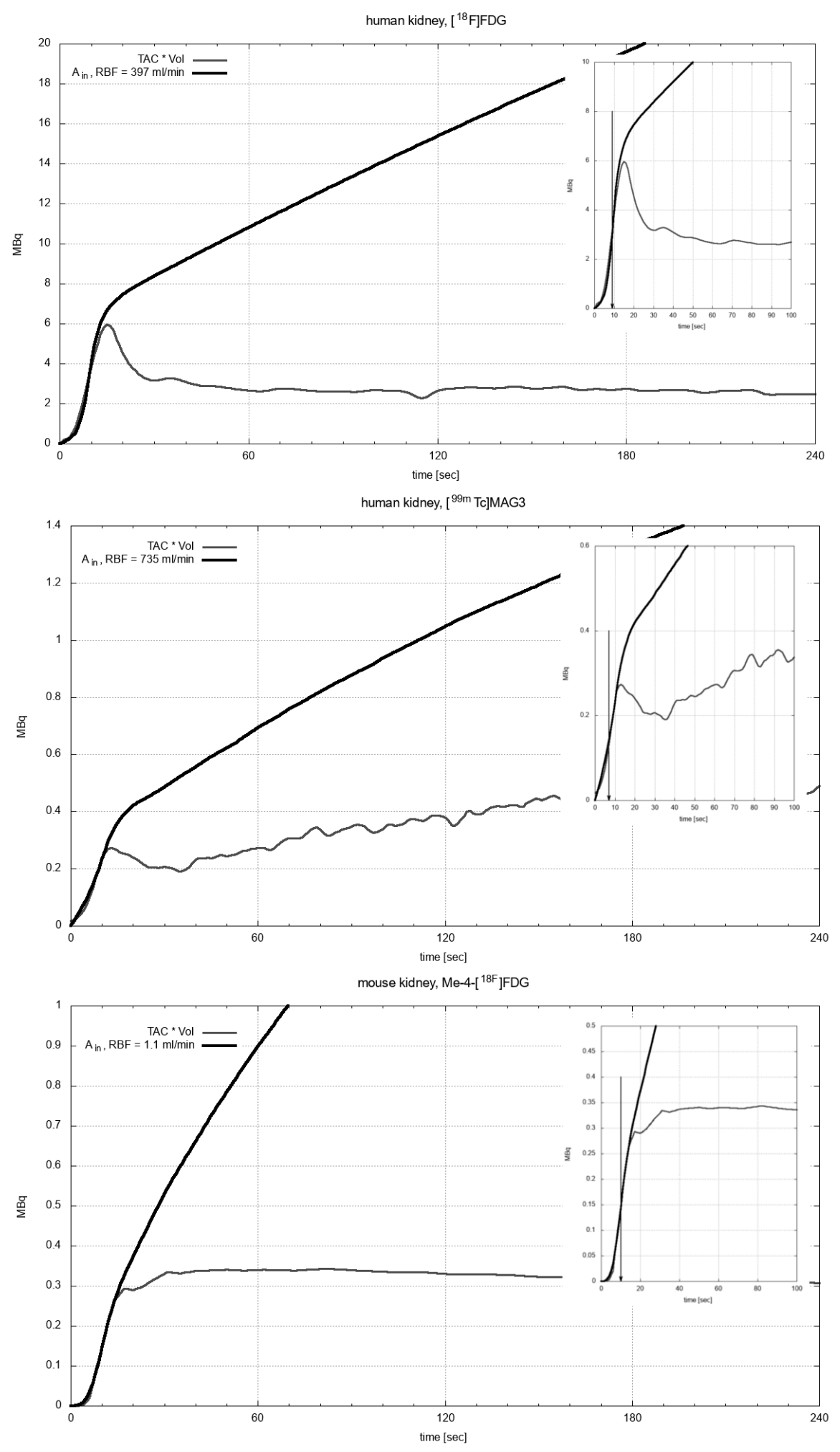

\section{Figure 1}

In gray, kidney time activity curves (TACs) are shown for three different radiotracers: human [18F]FDG (top), human [99mTc]MAG3 (middle) and mouse Me-4-[18F]FDG (bottom). The total amount of blood radiotracer concentration entering the kidney, i.e. the BF calculated from equ. (4) multiplied with $b(t)$, is displayed in solid black lines. The critical time point tcrit from which on the radiotracer starts to leave the kidney, the turning point, is indicated in the small images with an arrow. 

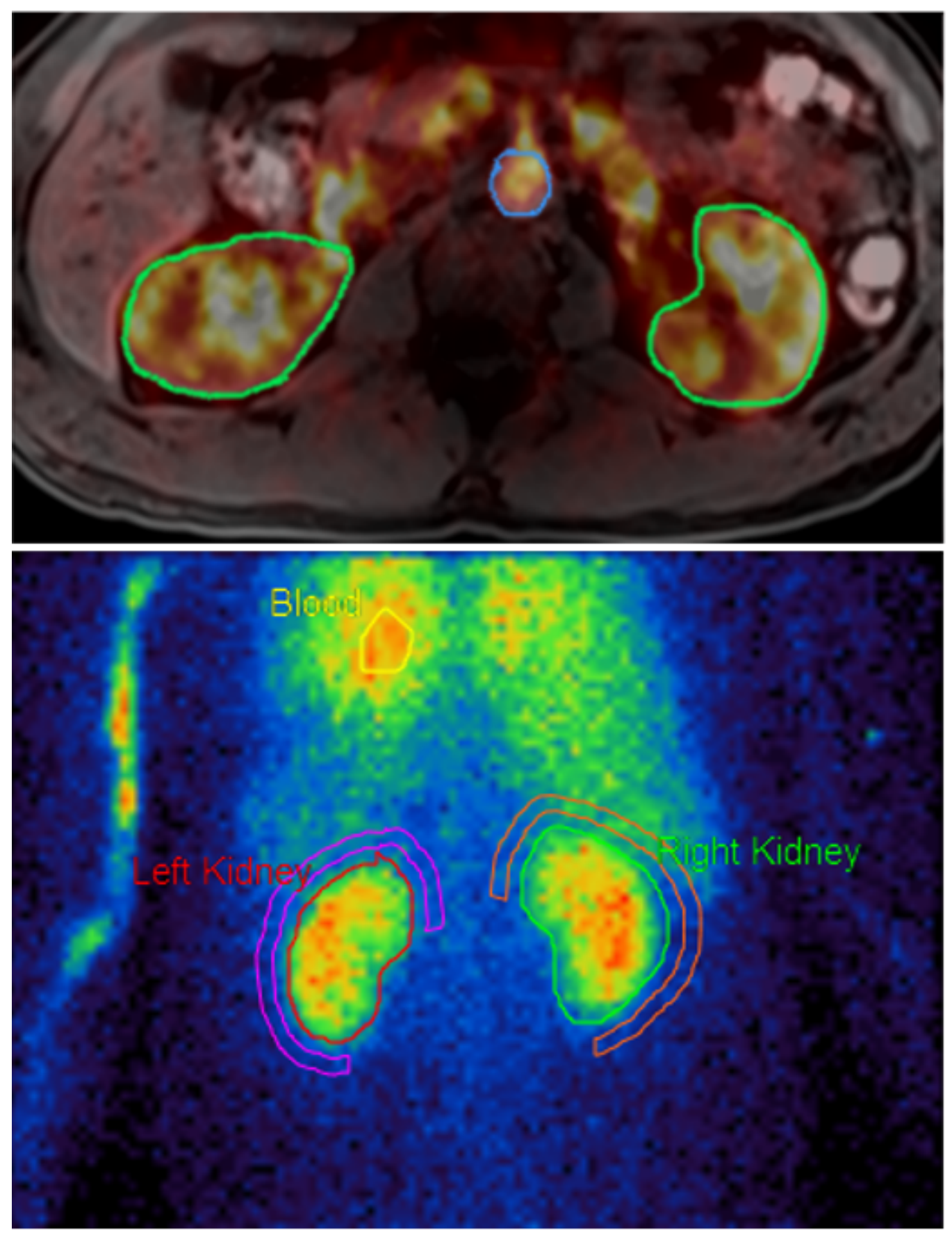

\section{Figure 2}

Top: human dynamic positron emission tomography and magnetic resonance imaging (PET/MRI) scan. Time activity curves (TACs) were obtained from volumes of interest (VOIs), which were delineated from the left and right kidney (green shapes), as well as from the aorta abdominalis (blue shape). Bottom: human dynamic renal scintigraphy. TACs were obtained from regions of interest (ROIs), drawn around the left and the right kidney as well as the left ventricle. 

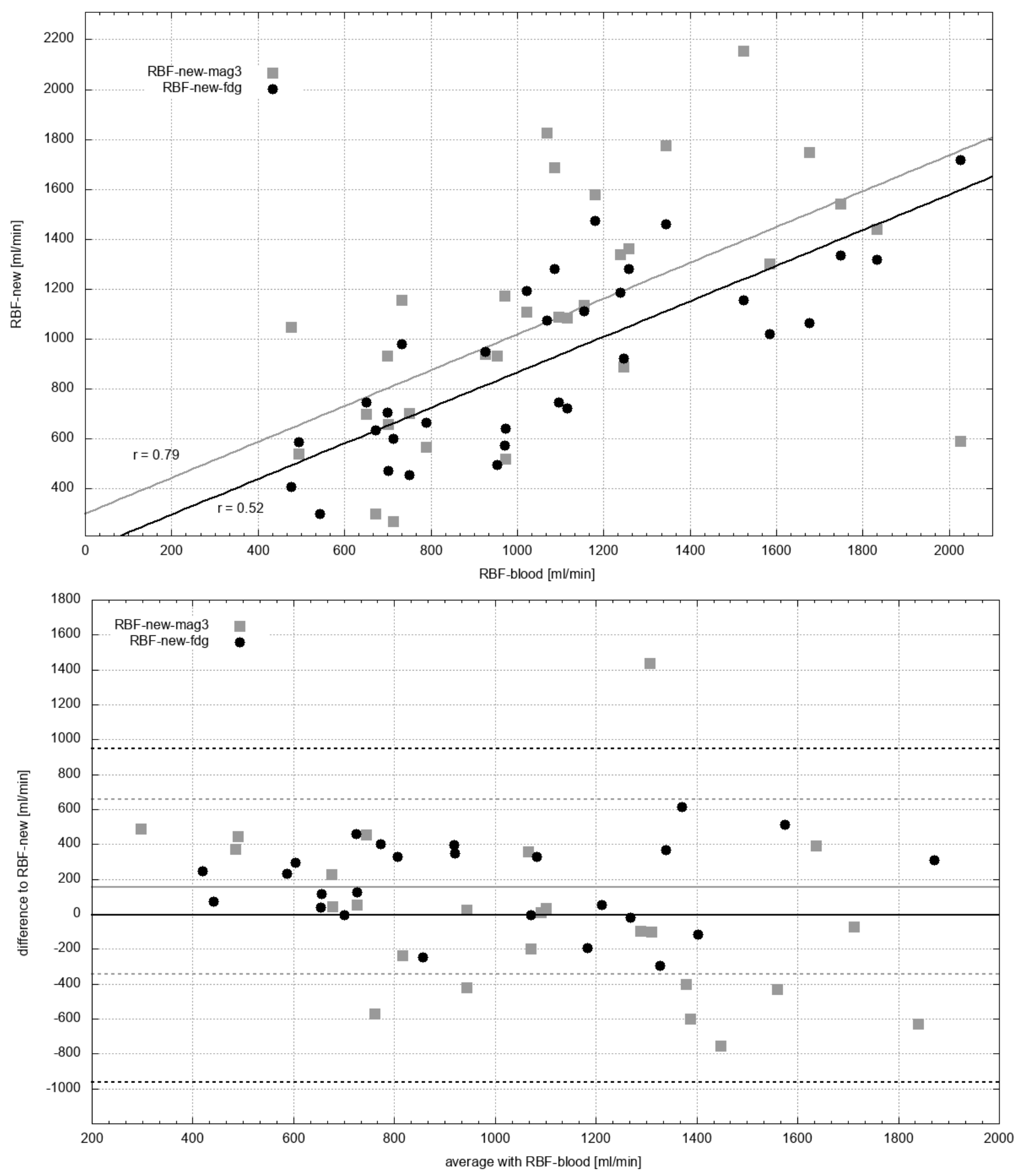

\section{Figure 3}

Top: RBF-new-FDG (black) and RBF-new-MAG3 (gray) versus the reference RBF-blood, lines of best fit are shown in the according color. Bottom: Bland-Altman analysis of RBF-new-FDG (black) and RBF-newMAG3 (gray). The difference mean value is indicated with the solid lines, plus and minus two standard deviations in dotted lines. RBF: renal blood flow. 